\title{
Corrugated Galvanised Iron Sheet as an Alternative to Conventional Roofing Materials "A case study from Sakteng Wildlife Sanctuary, Bhutan"
}

\author{
Sonam Tobgay, Kumbu Dorji \& Norbu Yangdon
}

Sakteng Wildlife Sanctuary, Trashigang, Department of Forests and Park Services, Bhutan

\begin{abstract}
Corrugated galvanized iron (CGI) sheet as an alternative to conventional roofing material was initiated under the integrated conservation development program (ICDP) of Sakteng Wildlife Sanctuary in the year 2004. The program was aimed at reducing community pressure on natural resources to enhance conservation of biodiversity and keystone fauna and flora species through protection of wild habitat. It has benefited 628 inhabitants of Merak and Sakteng which were identified as the least developed among 15 Gewogs (Blocks) under Trashigang Dzongkhag (District). Data were collected through semi structured questionnaires to study the impact of the program to policy and natural habitat management. Amongst the two types of conventional roofing materials available in the locality, Shingles (79\%) were in extensive use in comparison to Bamboo mat (21\%) attributing to its durability. However, people prefer to use CGI sheet $(100 \%)$ roofing which is economical, durable and effective in comparison to Shingle and Bamboo mat roofing, where incidences of water seepage to the room is frequent and requires replacing after 5.6 and 1.7 years respectively incurring higher maintenance costs. Replacing conventional roofing with CGI sheet has a long-term positive impact to conservation of forest and wild habitat. It is estimated that under ideal conditions approximately 5521 healthy matured Fir trees (>4'1"girth) and 1.68 million Bamboos were saved from felling since the initial supply of CGI sheet. However, effective roofing has led to the construction of the illegal permanent concrete huts in the Tsamdro (pastureland) and changes in the architectural designs of their native houses. The need of proper drainage system was also observed as important to reduce the gully erosion from CGI sheet roof run-off.
\end{abstract}

Keywords: Sakteng Wildlife Sanctuary, Merak, Sakteng, Shingle, Bamboo-mat, CGI Sheet

\section{Introduction}

Development and Conservation are two global and local objectives that can work against or with one another. Development is necessary for comfort and economic growth but on the other hand conservation is deemed equally important so that our future generations don't have to suffer due to current excessive nature exploitation in the name of development. To move development and conservation in tandem, Integrated Conservation Development Programs (ICDP) was designed.

Merak and Sakteng are the two remotest Gewogs (Blocks) of the Trashigang Dzongkhag (District) (Figure 1). Inhabitants are known as Brokpa and are transhumant by profession. Their livelihood is dependent on livestock rearing. Brokpas were deprived of developmental initiatives owing to their remote location that isolates them from rest of the country with the rugged mountains and harsh climatic conditions. 


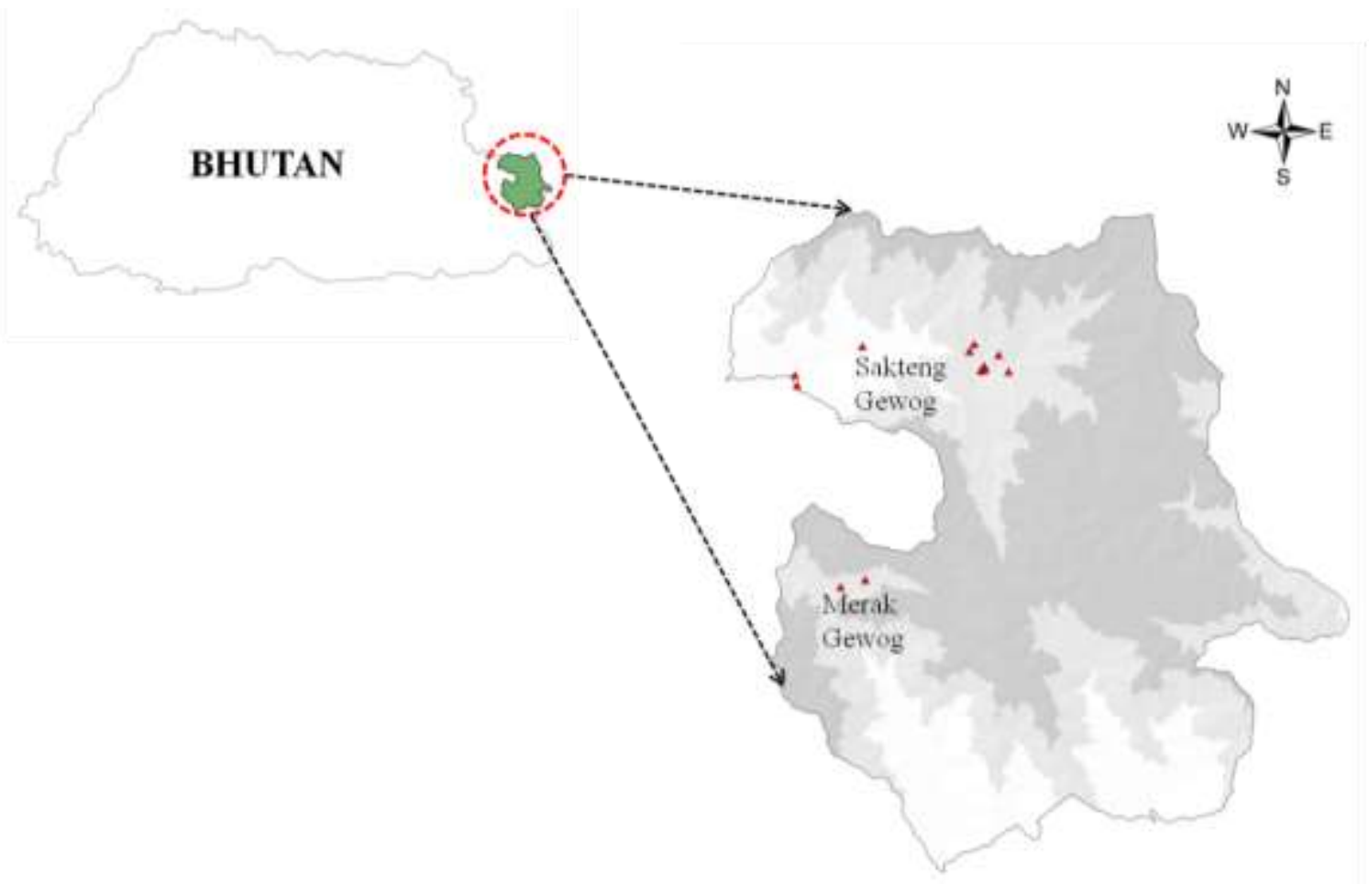

Figure 1 Study area map

Population growth over the last decades has increased pressure on forest and rangeland resources in Merak and Sakteng. In 2003, majority of the area under Merak and Sakteng Gewogs were declared as protected area with the establishment of Sakteng Wildlife Sanctuary (SWS) with the financial support from the Royal Government of Bhutan (RGoB) and World Wildlife Fund (WWF). From the inception of SWS, the natural resources management strategy and law enforcement regime has undergone transformation compared to the former territorial forest division. The newly established Sanctuary has initiated and focused on incentive based conservation approaches.

Through the approach of ICDP, the communities living in the Sanctuary were provided with environmental friendly alternatives to minimize the pressure on natural resources and compensate their sacrifices for conserving the natural biodiversity. Amongst various conservation related issues, extraction of huge amount of matured healthy trees and Bamboo for the production of Shingles and Bamboo mats for roofing were observed as a serious threat to the diverse ecosystems. The management of SWS with funding support from WWF has provided corrugated galvanized iron (CGI) sheet to 628 households of Merak and Sakteng on cost sharing basis ( $70 \%$ grant: $30 \%$ public collection) to minimize the mounting pressure of communities on natural resources. The CGI sheets were supplied in 2 phases: first phase in 2004 to 2005 and remaining in 2010 to 2011.

This study was carried out to assess the impact of replacing conventional roofing materials with CGI sheet under ICDP initiatives to the environment, livelihood and the community culture.

\section{Methodology}

\section{Data collection}

Out of the 628 benefited households from the CGI supply scheme, $63 \mathrm{HHs}$ were randomly interviewed taking 10 percent of the beneficiaries. Data were collected using a semi- structured questionnaire during the evening hours since people are usually engaged in their daily chores during the day. The questions comprised of: type of roofing materials, advantages and disadvantages, and pre and post scenario with the implementation of ICDP in the locality.

Age and gender of the respondents were taken in consideration to obtain quality information. Secondary data about the CGI supply scheme and 
number of beneficiaries were obtained from the ICDP records. Information on timber

demand and supply for roofing purposes were compiled from the forest information database (FID) maintained by the office of SWS.

\section{Data analysis}

Data was sorted and analyzed using MS-Excel 2007. Volume of the timber used for roofing was determined using the volume table provided by Forest Resource Management Division, Department of Forest and Park Services. Volume of the timber was calculated assuming timber with minimum girth size of $4^{\prime} 1 "$ as prescribed in Forest and Nature Conservation Rule (FNCR) 2006 [5]. Growth of the
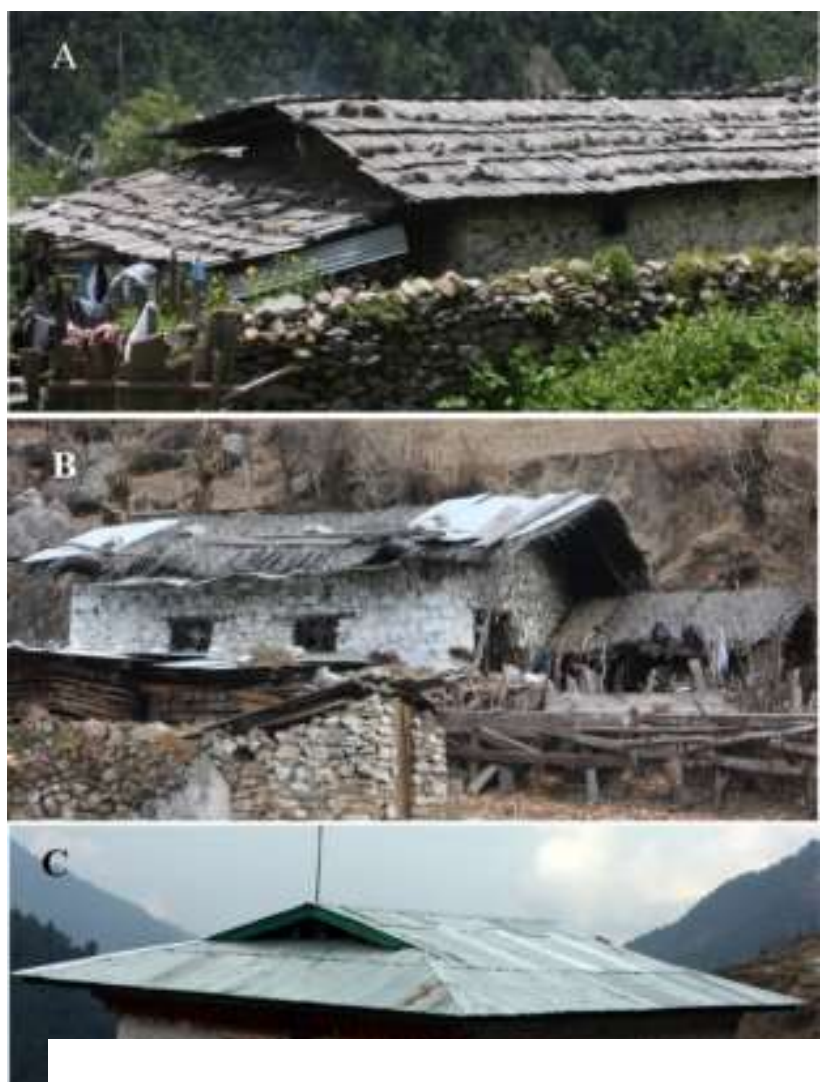

Figure 2 Types of roofing materials used in the study area (A)Shingles (B) Bamboo mats [Conventional] \& (C) CGI sheets.

\section{Impacts on natural resources}

A normal size house in the study area requires an average of 278 Bamboo poles or three mature tree ( $n=63$ ) with girth size 4'1" and above [5] to produce enough Bamboo mats and Shingles respectively for roofing. On average, Bamboo mat roofing has to be replaced every 1.7 years and Shingles roofing needs to be replaced every 5.6 years respectively. In the
Fir is determined using a girth (Girth= 2Pi*r). Following formula was used to study cost benefit of the particular scheme using Net Present Value of the investment:

$$
N P V=\sum_{t=1}^{T} \frac{C_{t}}{(1+r)^{t}}-C_{0}
$$

Where:

$\mathrm{C}_{\mathrm{t}}=$ net cash inflow during the period $\mathrm{t}$

$\mathrm{C}_{\mathrm{o}}=$ total initial investment costs

$r=$ discount rate, and

$\mathrm{t}=$ number of time periods

\section{Results and Discussion}

\section{Types of conventional roofing materials in the} study area

People of Merak and Sakteng Gewogs preferred Shingles and Bamboo mats as the roofing materials prior to the supply of CGI sheets. Fir Abies densa $[n=48]$ was the most used tree species for production of Shingles owing to its straight fiber pattern which enables easy shingle production. Bhutan pine Pinus bhutanica $[\mathrm{n}=2]$ and Bamboo Borinda grossa $[\mathrm{n}=13]$ were considered as an alternative source of roofing materials in places where Fir was limited or absent. A question on the usage of banana leaf, grasses and stone slabs for roofing were also included in the survey, however, none of the respondent talked about it mainly because of non-availability of such materials in the locality. Seventy nine percent of the respondents $(\mathrm{n}=50)$ were using Shingles and the rest $21 \% \quad(n=13)$ were using Bamboo as roofing materials (Figure 2). Shingles were in abundant use owing to its durability compare to Bamboo mat. Bamboo mats were used only in case of places where suitable Shingle producing tree species were not readily available in nearby locality. process of extracting the best raw materials, several associated trees and Bamboos are destroyed along with a huge quantity of off cuts left to decay in the forest.

Ideally, three mature trees are required to produce enough Shingles to roof a house but in reality people end up felling double ( $x$ 2) the number of required trees invariably in search of best quality tree free from heart rot or diseases. At times the felled tree doesn't serve the purpose of producing Shingle because of the defect in fiber pattern and one need to look for another healthy tree. In such process, a huge 
number of trees are exploited leading to massive forest destruction.

The first management plan of SWS revealed that 616 households residing in the park, on average constructed eight new houses per year and renovated 142 houses consuming 4188 trees. In addition, 257 healthy (straight fiber suitable for producing Shingles without heart rot) trees were felled only for roofing purpose [12].

Considering 15 years as the minimum lifespan of CGI sheet and the presence of 687 houses in Merak and Sakteng Gewog including 59 houses constructed after the issuance of CGI sheet, Shingle (5.6 years average lifespan) requirement for two Gewogs is estimated to be $8020.99 \mathrm{~m}^{3}$. Out of the total requirement, $7763.82 \mathrm{~m}^{3}$ is needed to meet the roof renovation and remaining $257.17 \mathrm{~m}^{3}$ to roof the 59 new houses.

As a result 5521 mature healthy Fir trees are required to be felled under ideal conditions (without taking account of other trees felled and damaged in search of suitable trees) to meet estimated Shingle demand of the locality.

With 1.7 years average life span of Bamboo mat roofing, it is estimated that 1.68 million Bamboo poles will be extracted. Further, the situation is worsened with dying of Bamboo from massive flowering since 2006. The study suggested that the cumulative radial growth of Fir under group opening and single tree selection was $36 \mathrm{~mm}$ and $14 \mathrm{~mm}$ respectively within 13 years [2]. Taking into account the suggested increment, Fir will take at least 70.4 and 181 years to reach the girth size of 4'1" (minimum girth of the trees to be allotted for Shingle production as per the FNCR 2006) under the respective silviculture system.

\section{Impacts on wildlife}

Forests of Merak and Sakteng are home to many endangered wild fauna species. Out of eight forest types [9] in SWS, Fir was recorded as one of the dominant tree species with Bamboo forming the understory in the mixed conifer forests, which is a potential habitat for Red Panda [1].

Amongst 37 faunal diversity in SWS [9], Red panda Ailurus fulgens prefers habitat with good Bamboo cover. Their primary diet consist of Bamboo leaves (50-100\%) supplemented by seasonal fruits and Bamboo shoots [7];[16]. Indiscriminate harvesting of Bamboo and timber besides grazing were identified as one of the most prominent threats for survival of Red panda [11]. Therefore, extensive extraction of the Bamboo for roofing purpose in the study area will directly pose a serious threat to their survival in the alpine ecosystem.

Comparatively, CGI sheet reflects sunlight more than the conventional roofing. Some of the beneficiaries $(n=9)$ have observed the decline in number and variety of avian fauna visiting their rooftop since the use of CGI roofing. They believe that it could be because of the glare and heat produced from the reflection of the unpainted CGI sheet roofing. Difficulty in nesting and few cases of causality of chicks due to skidding from the slippery CGI sheet were also observed by the respondents $(n=2)$.

\section{Policy Impact}

Ensuring environmentally sustainable future and maintaining 60 percent forest cover for all times is a massive policy commitment by the Royal Government of Bhutan [10]. Rapid economic development and increasing population are direct factors that will juxtapose the visionary pledge and lead to indiscriminate forest resources utilization and decrease forest cover below 60 percent. The only way forward to achieve this policy pledge is by

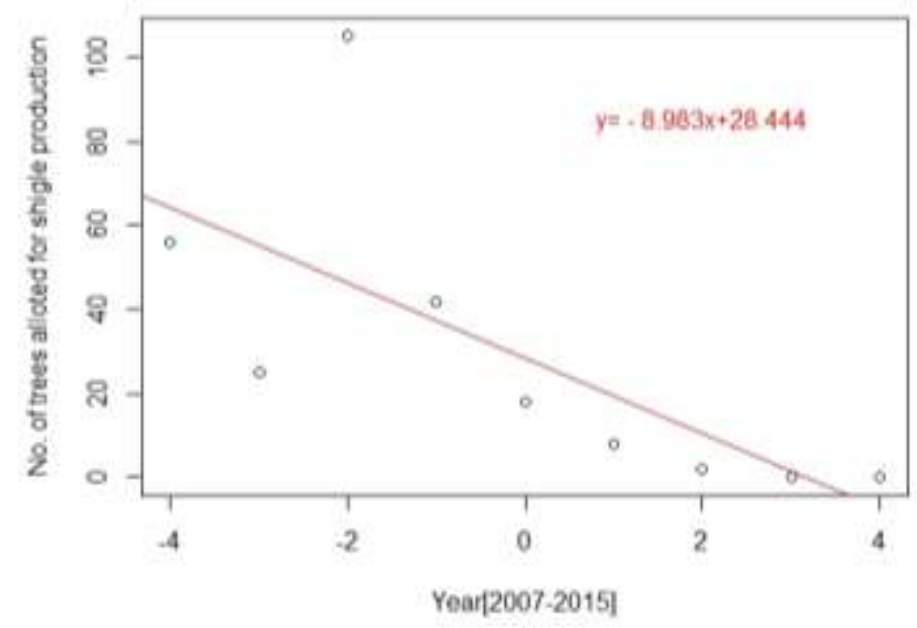

Figure 3 Shingle demand trend

gaining local stakeholders support through providing possible incentives.

Responding to this very important call, SWS with the financial support from WWF Bhutan has implemented the ICDP activities in Merak and Sakteng in financial year 2004-05 and 2010-11. 
This has reduced people's dependency on Bamboo and Shingles for roofing and also improved their support and participation in conservation initiatives. After the supply of the CGI sheet roofing, a drastic decline in demand for the Shingle was recorded with no demand since year 2014 (Figure 3).

On the contrary, this incentive has also led to the illegal encroachment of herders into the government owned pasture land (Tsamdro). Having provided more durable roofing materials, the Brokpas have started constructing concrete structures (permanent) in the pasture land which they do not own legally. Such illegal occupancy is more common at Cherbuling Tsamdro as reported by national print media [13] and may lead to conflict of interest losing the sole purpose of the conservation initiative.

Major changes in architectural design of the houses were also observed after the supply of CGI sheet roofing in the locality. Local people begun altering old design by adopting modern architectural design from neighboring lower valleys(Figure 4). Progressively, the inhabitants of Merak and Sakteng prefer the designs that are prominent in lower warmer valleys of Radhi and Phongmey (DrukZou: direct translation is Bhutan design) to their own unique design (Both zou means

\section{Social Impact}

The use of conventional roofing materials (Shingles and Bamboo mats) was laborious and less durable. On average, a household needs to spent 38.5 and 21.5 man-days to extract Shingles and Bamboo respectively to roof a house. Further, owing to less durability compared to CGI sheet the conventional roofing required frequent replacement leading to higher expenses on deployment of laborers and wastage of time.

CGI sheet roofing required higher initial investment but the cost is offset with better durability and less maintenance expenses in future. Therefore, it indirectly provides larger benefits in terms of time and monetary gains that can be invested to improve their livelihoods. With Nu.153.89/cft commercial rate of the Fir wood in $\log$ form (NRDCL, 2015) and 5.6 years average shelf life of the Shingle roofing against 15 years minimum shelf life of the CGI sheet roofing, every year timber worth of $\mathrm{Nu} 1.81$ million is saved from being felled with annual discounting rate of 5 percent.
Tibetan design). When people were questioned on reasons for the change in housing designs, $34.9 \%$ of the respondents attributed the change to effective roofing from the CGI sheet, exposure (31.7\%), comfort $(28.5 \%)$ and social status $(4.7 \%)$.

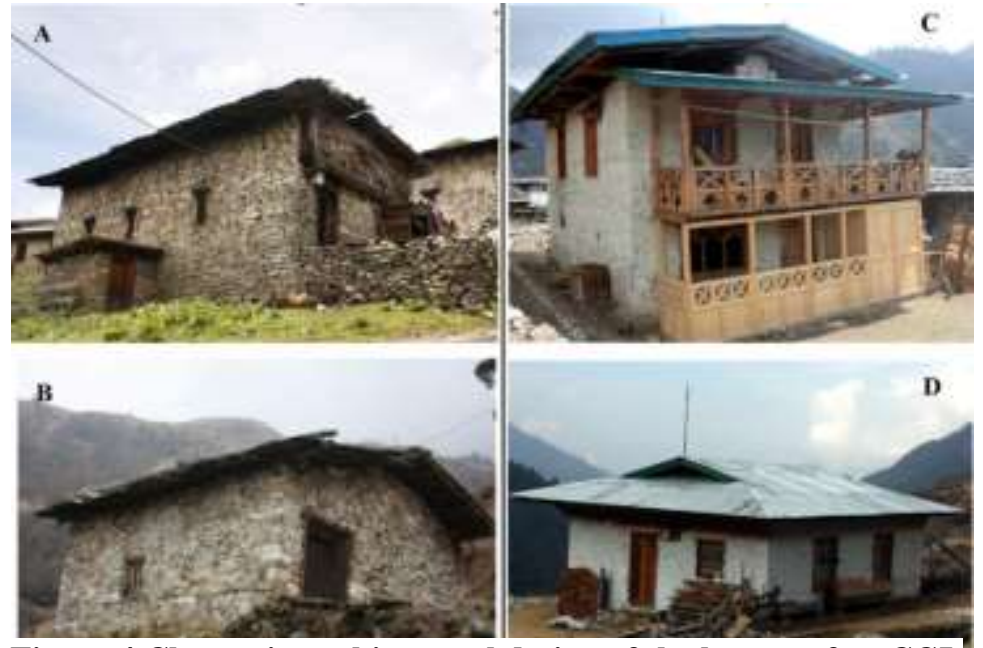

Figure 4 Change in architectural design of the houses after CGI sheet roofing $(\mathrm{A}) \&(\mathrm{~B})$ Conventional and $(\mathrm{C}) \&(\mathrm{D})$ preferred.

Therefore, it was found that without the proper policy in place, incentivizing people with CGI roofing possesses an adverse impact to the traditional design in the locality.

Dirtying of rooms and damage of the properties from water/rain seepage during heavy rain and snow accumulation was recorded as very common $[n=62]$ with conventional roofing (Figure 5). Meanwhile the supply of CGI sheets improved effectiveness of roof against water seepage, which directly helped in improvement of household hygiene of the locality.

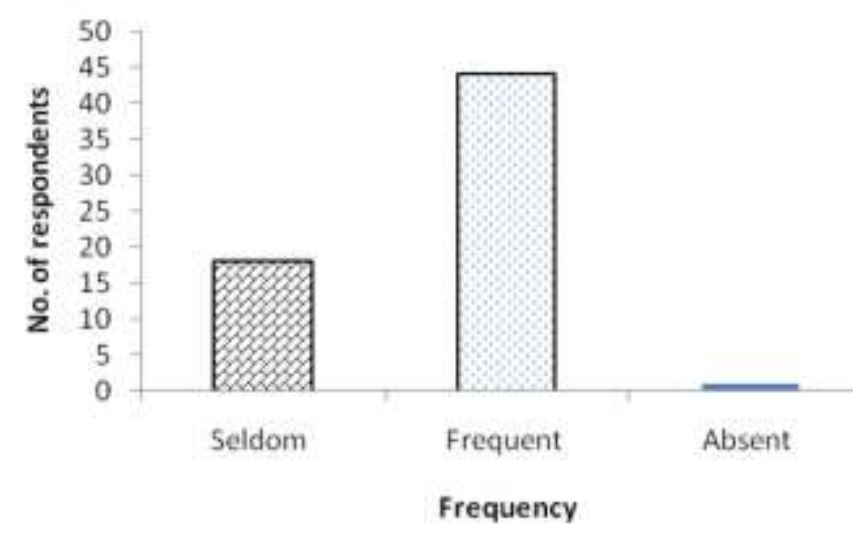

Figure 5 Incidence of water seepage in conventional roofing 
Replacing Shingle roofing with CGI sheet using the traditional roof frame (non engineered structure) method was known to result in pullthrough failure of connections incidences [4]; [8] and was recorded as a prime reason for major windstorm damage in rural areas in Bhutan [4]. Similarly, houses of Merak and Sakteng were roofed with CGI sheet on conventional roof frames, which makes them vulnerable to windstorm damage.

\section{General Impacts}

Soil: Sakteng and Merak have poor drainage systems because of clustered settlements. Narrow footpaths between adjacent houses create erosion. Under such conditions, there were no proper drainage to channel the rain water collected from CGI roofing during intensive rainfall which results in heavy surface runoff within the village, later forming a huge gully right below the settlement area.

Aesthetic: Conventional roofing perfectly blends with the color of the natural environment which complements the beauty of nature unlike CGI sheet roofing. Intensive reflection of sunrays from the CGI roofing amidst the peaceful valleys looks alien to the outside spectator. It looks more or less like a booming mini town instead of a well known culturally unique and beautiful Brokpa settlement in the alpine meadow (observation and comments of the tourist).

Microclimate: Several respondents $(n=11)$ were positive that they have experienced more heat since the arrival of CGI sheet. They considered that the increase in local heat to be the result of intensive reflection of sun rays from the unpainted CGI sheets.

\section{Conclusion and Recommendations}

In this study, the impacts of replacing conventional roofing with CGI sheet to the environment, social, policy and traditional practices were assessed. CGI sheet roofing was found to possess positive impact in terms of forest, natural resources and wild habitat management. However, it has adverse impact to the unique Brokpa culture and tradition. Based on the findings, the following recommendations are suggested:

\section{Paint CGI roof with environmental friendly colours to maintain aesthetic looks}

Painting of the roof with environmental friendly color paint is recommended as a viable option to overcome the issue of maintaining aesthetics and reducing glare, which is suspected to interfere with life of avi-fauna. Further painting of CGI roofing with a fine film of suitable paint is known to prolong the durability of roof even when considerable amount of the zinc coating has been lost from the sheet [6].

\section{Protection and prevention of gully formation}

A gully in the study site was formed from an increase in surface run-off as a result of improper drainage management. Improvement of gully catchments to reduce and regulate the run-off rates, diversion of surface water and stabilization by structural measures accompanying revegetation are some of the suggested gully controlling measures [3]. Therefore, constructing proper drain networks along the periphery of footpaths within the village and a few check dams along the gully is recommended to prevent further degradation.

\section{Preservation of local housing designs to conserve aged old culture and tradition}

The majority of the houses in the study area have undergone change to suit the present life style of the families and only a few houses are left without much change. One house each in Merak and Sakteng could be preserved as a cultural display centre as a part of promotion and preservation of local culture and tradition.

\section{Preparation against windstorm hazards}

Providing strong support string, gable end stiffener, bracing and sand bags (10-20kgs) over the roof cladding is recommended to prevent the uplift of roof [4].

\section{Acknowledgements}

The authors sincerely acknowledge the field staff of Sakteng Wildlife Sanctuary for helping to collect the data and Mr. Thinley Wangdi, Park Manager for his support and guidance.

Authors are also sincerely thankful to Dr. Joanne Millar, Senior research fellow, Charles Sturt University for her valuable edits and inputs in finalizing the paper. 


\section{Reference}

1. Dorjee, K., \& Katel, O. N. (2012). Transhumant Practices and its impact on Red Panda Habitat in Sakteng Wildlife Sanctuary, Bhutan. Sakteng Wild life Sanctuary, Sakteng, Trashigang, eastern Bhutan.Unpublish

2. Dukpa, D., Khandu, Y., Darabant, A., \& Cook, E. R. (2012). Growth release of retained fir (Abies densa) trees in the group opening and single tree selection logging. Bhutan Journal of RNR, 8 (1), 113-119..

3. Geyik, P. M. (1986). FAO watershed management field manual- Gully control, FAO Conservation guide 13/2. Rome: Food and Agriculture Organization of United Nations

4. Lotay, Y. (2015). Wind induced Damage to roofs, A comparative study on roofs in Bhutan and Japan. Asian Disaster Reduction Center.

5. Ministry of Agriculture. (2006). Forest and Nature Conservation Rules of Bhutan. Department of Forest, Royal Government of Bhutan.

6. N.S.W Heritage Office. (2004). Corrugated roofing. Endorsed by the Heritage Council of NSW Technical Advisory Group, Australia ISBN 1876415789.

7. Pradhan, S., Gautom, K. S., \& Jamal, A. K. (2001). Ecology of the red panda Ailurus fulgens in the Singhalila National Park. Biological Conservation, 98, 11-18.

8. Ramli, N. I., Majid, T. A., Chik, F. A., Muhammad, M. K., \& Deraman, S. A. (2014). Assessment of Pull-through Failure of Nail Connection for Rural
Roofing System under Wind Load in Malaysia. Journal of Civil Engineering Research, 4(3A):, 135-137.

9. SWS. (2016). Conservation Management Plan (2017-2024), Sakteng Wildlife Sanctuary. Department of Forests of Park Services, Royal Governmentof Bhutan; and Bhutan Trust Fund for Environmental Conservation, Thimphu, Bhutan.

10. The Constitution of the Kingdom of Bhutan. (2008). Article 5, Section 3

11. Wangchuk, K. (n.d). Habitat status and conservation threats of the Red Panda in Jigme Singye Wangchuck National Park. The Rufford Foundation.

12. Wangchuk, S. (2008). Management Plan for Sakteng Wildlife Sanctuary. Ministry of Agriculture, Department of Forest. Royal Government of Bhutan.

13. Wangdi, T. (2015, April 29). Illegal occupancy in Merak. http://www.kuenselonline.com/illegaloccupancy-of-in-merak/, p. 6.

14. World Wildlife Fund. (2015). Sakteng Wildlife Sanctuary. http://wwf.panda.org/who_we_are/wwf_off ices/bhutan/projects/index.cfm? uProjectID $=$ BT0874.

15. World Wildlife Fund\&Sakteng Wildlife Sanctuary(2011). Participatory Zoning for Sakteng Wildlife Sanctuary;Balancing Conservation and Development Goals. Department of Forest and Park Services , World Wildlife Fund Bhutan and Sakteng Wildlife Sanctuary. Ministry of Agriculture and Forest.

16. Yonzon, P. B., \& Hunter, M. L. (1991b). Conservation of the red panda Ailurus fulgens . Biological Conservation, 75, 111. 